\section{Pandemia COVID-19: repercusiones en la educación universitaria}

\section{COVID-19 pandemic: impact on university education}

\section{Sr. Editor.}

A partir del mensaje del presidente de la República del Perú y de la declaratoria del estado de emergencia, el 16 de marzo del $2020,{ }^{1}$ que incluía cierre de fronteras, como primera medida, frente a la crisis mundial desatada por la pandemia del coronavirus, ${ }^{2}$ hizo que nuestras vidas y hábitos cambien de forma rápida y radical con la finalidad de salvaguardar la salud con la consigna "quédate en casa", situación que dejó al descubierto deficiencias no sólo en el sector salud, situación que era ya visible, sino también en uno de los pilares del desarrollo de un país, la educación. ${ }^{1}$

Al igual que en todo el mundo el COVID-19 cambió la vida de más de 33 millones de peruanos conllevando a una crisis en la educación superior universitaria con impacto de más del $87 \%$. Las universidades en el Perú tuvieron que cerrar sus puertas, las clases de ciclos vacacionales interrumpidas, los estudiantes y docentes que cursaban pasantías e intercambios a nivel nacional e internacional fueron cancelados al no poder ser evaluados y por tanto con dificultad para retornar a sus lugares de origen. ${ }^{3}$ Las implicancias inmediatas corrían al ritmo del aumento de casos por coronavirus, pero cuáles serían las repercusiones a mediano y largo plazo, la respuesta de la Superintendencia Nacional de Educación Superior (SUNEDU) predijo ser positivas. ${ }^{4}$

Ante el incremento de semanas al estado de emergencia, SUNEDU y MINEDU (Ministerio de Educación), autorizan el inicio de actividades universitarias vía online, incentivando a toda la comunidad universitaria tomar énfasis en programas y estudios de investigación dirigidos al COVID-19. Esta situación hizo poner en marcha plataformas virtuales con que cuenta toda universidad peruana licenciada como requisito de uno de los estándares de calidad. ${ }^{4}$

Frente a ello el 04 de abril de 2020, se reunieron los 35 rectores adscritas a la Asociación Nacional de Universidades Públicas del Perú (ANUPP), sobre las problemáticas y limitaciones de cada institución, dando a conocer el inicio irremediable de clases el 04 de mayo, el mismo que se realizará de manera progresiva y por etapas, de manera que al llegar a ser presencial, se respetará el aforo en las aulas para evitar contagios por coronavirus, medida refrendada mediante RVM N. ${ }^{\circ} 084-2020.5$

La Universidad de San Antonio Abad del Cusco (UNSAAC), puso en marcha la reactivación de la plataforma virtual Classroom, indicando a autoridades, docentes y estudiantes entrar en capacitación urgente, toda vez que a través de un formulario llenado por todos los involucrados,

\section{Carta al Editor}

Lida Velazque Rojas ${ }^{1, a}$, César Joe Valenzuela Huamán 1,a Fernando Murillo Salazar ${ }^{1, b}$

${ }^{1}$ Universidad Nacional de San Antonio Abad del Cusco, Facultad de Ciencias de la Salud, Cusco, Perú.

a Doctor en Ciencias de la Salud.

${ }^{\mathrm{b}}$ Doctor en Salud Pública.

\section{Correspondencia:}

Lida Velazque Rojas: lidacionn@hotmail.com / lida.velazque@unsaac.edu.pe Av. De la Cultura N 1400-b, oficina 204, segundo piso, esquina con pasaje M. Carrasco. ORCID: 0000-0003-1219-7456

\section{Coautores:}

César Joe Valenzuela Huamán: qf.joes@gmail.com ORCID: 0000-0002-1158-6233

Fernando Murillo Salazar: fermusa666@gmail.com

Conflicto de intereses: los autores refieren no tener ningún conflicto de intereses

Recibido: $16 / 03 / 20$

Aceptado: 23/03/20

Publicado: 09/05/20 
se notaron deficiencias en su aplicación, principalmente del docente universitario, observándose en todo el proceso extremos de perfección y deficiencia. Se iniciaron reuniones de videoconferencias con docentes y autoridades de cada facultad para tomar medidas estratégicas para el semestre 2020-1, debiendo incluir ésta, clases virtuales, semipresenciales y presenciales a posteriori, apostando por la salida de la crisis, que incluyó entrega de informe a SUNEDU y MINEDU. Todo este proyecto inicial fue posteriormente refrendado en un plan de trabajo que contiene cuatros ejes estratégicos, la primera direccionada a la reprogramación de las actividades académicas, que incluye matrícula de estudiantes, instalación de plataforma virtual de acceso a estudiantes y docentes, recopilación y validación de firmas virtuales de autoridades de cada facultad, calendarización de asignaturas no presenciales y presenciales. El segundo eje dirigido a la gestión de adaptación no presencial de las asignaturas que incluiría la elaboración de documentos de gestión académica adaptados a educación no presencial como reglamentos, directivas, guías, sílabos, entre otras; además se determinará la relación de asignaturas de pre y posgrado a ser impartidas en forma no presencial y presencial, uso de tecnologías de información y comunicación, conectividad y soporte administrativo de la UNSAAC de acuerdo a las necesidades, demandas y requerimientos del proceso académico y principalmente a realizar capacitación a docentes y estudiantes en educación a distancia con entornos virtuales.

Los ejes tercero y cuarto, serán destinados a realizar seguimiento y acompańamiento a docentes y estudiantes en cuanto se ejecuten el inicio de labores y a mitigar riesgos en toda la ciudad universitaria, tratando de encontrar aliados estratégicos locales con el fin de mejorar la calidad de enseñanza y aprendizaje.

Desde ya, esta drástica decisión, no intencional, va produciendo un impacto positivo en este nivel educativo, aun siendo pasajera, abrió expectativas de mayor comunicación entre docentes y estudiantes para utilizar la tecnología como herramienta de desarrollo en ambos grupos, modelo al que se temía llegar y depender, en las que muchas veces fue sinónimo de retraso en nuestra ciudad universitaria por el escepticismo de muchos.

No se puede negar las limitaciones que se presentarán, por lo complicado que resulta para muchos docentes realizar cursos a distancia y de manera fluida, efectiva e interactiva, especialmente visible en las universidades públicas del Perú. Son pocas las instituciones que aplican un modelo educativo en línea y éstas van direccionadas principalmente a escuelas de posgrado, donde los cursos a distancia son prioridad. Por otra parte, se considera también las limitaciones tecnológicas que pueda presentar el estudiante, considerando que nuestra universidad es una institución pública que alberga a estudiantes en su mayoría de provincias y de nivel socioeconómico bajo y medio, teniendo como mínimo sólo los servicios básicos, no contando todos ellos por ejemplo con una computadora o servicio de internet eficiente y continuo o tal vez de espacios físicos disponibles para trabajar desde su vivienda, más aún en aquellos estudiantes que reciben apoyo de la universidad como es el comedor universitario y el internet gratuito, disponible en el campus, conllevando estos aspectos a disminuir, de inicio, la accesibilidad a la educación.

La mayoría de escuelas profesionales podrán adaptarse a este sistema, pero hasta ahora el talón de Aquiles de muchas universidades, es como adaptar por ejemplo las escuelas profesionales de Odontología y Medicina, donde los estudiantes realizan prácticas de rotación adaptadas a hospitales. Odontología aún es más preocupante, la mitad de la malla currícular de estudios es desarrollada a través de prácticas docentes guiadas, direccionadas únicamente a la atención del público en general, cumpliendo un récord de tratamientos en cada área y especialidad dentro de una Clínica Odontológica. Se ha observado ante ello, que muchas universidades extranjeras en Espa$\tilde{n} a^{23}$, México y Chile abordaron de inicio suplir las prácticas clínicas por reforzamientos teóricos virtuales de las áreas. Este modelo planteado fue utilizado en la UNSAAC en el semestre 2018-2, por problemas suscitados dentro de la Clínica Odontológica, en la que los cursos clínicos fueron desarrollados de manera presencial como reforzamiento teórico y resolución de casos clínicos y en la medida que fue posible, utilizando laboratorios de cada área, así pudimos evaluar en el 2019, que los estudiantes de esta escuela profesional necesitan realizar tratamientos en pacientes; una maqueta o simuladores no pueden suplir las complicaciones y dificultades que el ser humano, propiamente puede presentar, como resultado informamos disminución en la calidad y cantidad de atenciones. Frente a ello, querer imponer como alternativa a éstas escuelas profesionales un manejo virtual teórico de lo que es netamente clínico, conllevará a problemas mayores y serios en la formación de profesionales que necesiten la parte de laboratorio presencial.

Por otra parte, se hace necesario e inmediato crear protocolos de atención para cada área y especialidad de la Odontología de manera que sea aplicable en todas las universidades del Perú en situaciones como la que estamos viviendo, y más aun siendo estas netamente presenciales.

Sin embargo, como todas las crisis pasadas en el mundo, la pandemia del coronavirus también será parte de nuestra historia, donde existirá un antes y un después, pero también será un despertar para planear y mejorar a largo plazo la internacionalización de la educación superior universitaria a través de cursos en línea, utilizando las plataformas virtuales de forma efectiva y principalmente deje de ser un sector olvidado para el estado, invirtiendo en tecnología y en el potencial humano universitario.

\section{Referencias bibliográficas}

1. El Peruano [Internet]. Lima: Decreto Supremo que declara Estado de Emergencia Nacional por las graves circunstancias que afectan la vida de la Nación a consecuencia del brote del COVID-19. 2020 [citado el 10 de abril del 2020]. Disponible en: https://busquedas. elperuano.pe/normaslegales/decreto-supremo-que-de- 
clara-estado-de-emergencia-nacional-po-decreto-supremo-n-044-2020-pcm-1864948-2/

2. OPS/OMS [Internet]. La OMS caracteriza a COVID-19 como una pandemia. 2020 [citado el 3 de abril del 2020]. Disponible en: https://www.paho.org/ hq/index.php?option $=$ com_content\&view $=$ article\&id=15756:la-oms-caracteriza-a-covid-19-comounapandemia\&catid $=740 \&$ lang $=$ es $\&$ Itemid $=1926$

3. UNESCO/IESALC [Internet]. El coronavirus COVID-19 y la educación superior: Impacto y recomendaciones. 2020 [citado el 10 de abril del 2020]. Disponible en: https://www.iesalc.unesco.org/2020/04/02/el-coronavirus-covid-19-y-la-educacion-superior-impacto-y-recomendaciones/

4. El Peruano [Internet]. Criterios para la supervisión de la adaptación de la educación no presencial, con carácter excepcional, de las asignaturas por parte de universidades y escuelas de posgrado como consecuencia de las medidas para prevenir y controlar el COVID-19. 2020 [citado el10 de abril del 2020]. Disponible en: https://busquedas.elperuano.pe/normaslegales/aprueban-los-criterios-para-la-supervision-de-la-adaptacion-resolucion-n-039-2020-sunedu-cd-1865206-1/
5. La República [Internet]. Asociación Nacional de Universidades Públicas del Perú (ANUPP). Perú 2020 [citado el 4 de abril del 2020]. Disponible en: https://arepublica. pe/sociedad/2020/04/03/estado-de-emergencia-universidades-publicas-afiliadas-a-la-anupp-acuerdan-reducir-el-aforo-de-aulas-ante-reanudacion-de-clases-atmp/

6. Giner-Tarrida L. El coronavirus y la docencia en la universidad. Dental tribune [Internet]. 2020 [citado el 4 de abril del 2020];17(4):8. Disponible en: https://la.dental-tribune.com/epaper/dental-tribunes/dt-latin-ameri$\mathrm{ca} / \mathrm{dt}$-latin-america-no-4-2020.pdf 
